\title{
Les muscles de la tête du chameau
}

\author{
par M. A.F. TAYEB, M. V.SC. \\ Ccliege of veterinary medicine, Fouad Ior. Cairo (Egypt)
}

\section{INTRODUCTION}

LESBRE (1903) signale la présence d'un muscle lacrymal et d'un muscle frontal chez le chameau. Le releveur commun de l'aile du nez et de la levre supérieure forme une mince et vaste expansion qui s'unit au lacrymal et couvre complètement le canin, sans être perforé par lui. Le canin résume à la fois le canin et le releveur propre de la lèvre supérieure du cheval et s'unit à celui du côté opposé.

Le muscle zygomatique est allongé et se perd en avant sur le buccinateur à 10 centimètres de la commissure des lèvres. Le buccinateur est un muscle ample, recouvert le long de son bord inférieur par les glandes buccales.

Lesbre rapporte également la présence du crotaphite et montre que les fibres de la couche superficielle du masséter forment un angle de 30 à 40 degrés avec le grand axe de la tête.

Le ptérygoìdien interne est bien développé; le ptérygoïdien externe n'est pas distinct.

Le dilatateur des narines (transversal du bout du nez) et le nasal semblent faire défaut.

Le digastrique ressemble à celui du cheval; le mylohyoïdien est mince; le génioglosse est épais; le stylohyoidien est allongé; l'occipitohyoïdien se confond avec le digastrique.

Lesbre rapporte l'absence des muscles cervicozuriculaire et tympano-auriculaire.

L'objet de cet article est de décrire dans le détail les muscles de la tête du chameau.

\section{MATÉRIEL D'ÉTUDE ET MÉTHODE}

Les animaux de dissection furent pris aux abattoirs du Caire. On préleva 10 têtes de chameaux soudanais des deux sexes; les têtes furent sectionnées en arrière de la deuxième vertebre cervicale, puis injectées par l'artère carotide avec une solution de formol à $10 \%$.

\section{LES MUSCLES}

\section{DE LA TÊTE DU CHAMEAU}

Ils comprennent :

Les muscles des lèvres et du nez, des joues, les

\author{
Traduction : P.-C. BLIN
}

Chế de Travaux d'Anatomie à l'École Nationale Vétérinaire d'Alfort

muscles des paupieres, les muscles des mâchoires et les muscles auriculaires. Les muscles de la tête sont recouverts par la couche continue du peaucier de la face. Celui-ci provient de la couche superficielle du muscle sterno-mastoïdien (sterno-céphalique) et du muscle omo-hyoïdien. La large nappe du peaucier s'étale sur la partie inférieure du masséter et le bord postérieur du maxillaire inférieur, s'épaissit progressivement en devenant plus étroite en avant pour former un risorius ample, épais, allongé, s'insérant à la commissure des levres.

Contrairement à ce que dit Lesbre, le zygomatique n'est recouvert par le buccinateur que sur 3 à \& centimetres à partir de la commissure des lèvres. Le buccinateur rappelle quelque peu le platysma de i'Homme. A son bord supérieur, une très mince nappe musculaire se dirige vers le haut pour se confondre avec le muscle zygomatique; à partir de celui-ci, elle gagne la face dorsale de la tête pour se cerdre dans les muscles de cette région.

Le risorius recouvre la terminaison de la veine faciale, la veine labiale inférieure et le nerf buccal inférieur.

\section{Muscles des lèvres, du nez, des joues.}

L'orbiculaire des lèvres. - Bien qu'il soit épais et bien developpé, il ne forme qu'un anneau incomplet par suite de la fente profonde qui divise la lèvre supérieure en deux moitiés susceptibles de se mouvoir isolément.

Le releveur commun de l'aile du nez et de la lèvre supérieure. - C'est un muscle d'épaisseur moyenne, aplati, étendu, se confondant en arrière avec le malaire (toutefois, on les sépare aisément avec le scalpel), pour former un sus-naso-labial très développé s'étendant amplement sur le côté de la face. Le tendon d'origine du releveur commun est mince; il s'insère sur les os nasal et frontal; le releveur commun se confond avec son homologue du côté opposé. Arrivé sur le maxillaire supérieur, le tendon du releveur commun donne suite à une lame musculaire mince dont les fibres divergent pour s'insérer respectivement dans la lève supérieure et les naseaux. Ce muscle recouvre entierement le canin sans être pénétré par lui, comme c'est le cas chez le cheval. 
Le malaire (lacrymal de Lesbre). - Ce muscle s'étend sous forme d'une couche ample et épaisse, de la commissure des lèvres en avant jusqu'au milieu de la face externe du masséter en arrière. Il est entièrement sous-cutané, sauf une étroite bande longitudinale qui est cachée par le zygomatique. En haut, le muscle prend son insertion sur le maxillaire superieur, le lacrymal et le zygomatique, en se confondant avec l'orbiculaire des paupières.

En avant et en bas, il se confond avec le buccinateur, mais en arrière et en bas il se mélange aux fibres du mince peaussier de la face qui recouvre le masséter.

Le zygomatique. - Il se présente comme un muscle rubané, long, épais, large de $2 \mathrm{~cm} .5$ environ, s'étendant de la crête zygomatique où ił prend origine, comme d'ordinaire, à partir du fascia qui recouvre le masséter, associé à un mince tendon nacré procédant de la crête zygomatique elle-même.

Sur 3-4 centimètres précédant son insertion à la commissure des lèvres, le zygomatique est masqué par le buccinateur (et non sur 10 centimètres, comme le précise Lesbre). Le muscle zygomatique est en rapport en dedans avec le masséter, le malaire, la veine faciale, le nerf buccal supérieur et une partie du canal de Sténon.

Le canin. - $\mathrm{Ce}$ muscle combine dans une certaine mesure l'action du nasal et celle du releveur propre de la lèvre supérieure du cheval.

L'élévation extrême de la lèvre supérieure (pour découvrir la muqueuse), comme c'est le cas chez les Équidés, ne se fait pas chez le chameau. Le canin s'attache par des tendons nacrés sur une ligne d'insertion surplombant la premiere dent molaire et la dépassant légèrement en avant. Ces tendons sont suivis par une portion charnue, dont les fibres, d'abord d'aspect torsadé, se déploient en éventail jusque dans les naseaux.

Un faisceau cylindrique et épaissi de ce muscle s'étend en avant sous l'aile externe des naseaux pour s'insérer en partie antérieure de la lèvre supérieure dans la moitié correspondante de celle-ci. Les fibres de ce faisceau tendent à se réunir à celles du faisceau opposé, en s'incurvant vers le bas, les unes à la rencontre des autres sans se pénétrer (contrairement à l'opinion de Lesbre qui veut qu'elles s'unissent de manière à se mélanger).

Le canin est complètement recouvert par le susnaso-labial, mais il est en rapport, en profondeur, avec le tendon nasal du buccinateur, le nerf et l'artère infraorbitaires.

Le buccinateur. - La portion antérieure de la couche superficielle de ce muscle est formée de fibres constituant un massif épais et continu dirigées verticalement; le raphé médian fait défaut. En haut, les fibres prennent naissance sur la face externe du maxillaire supérieur, à șa jonction avec l'os nasal et l'apophyse nasale de l'intermaxillaire, par un tendon nacré demi-circulaire qui se dégage sous le canin et fait place aux fibres inférieures du buccinateur qui recouvrent les insertions du risorius et du zygomatique. La portion postérieure de la couche superficielle est plus mince, faite de fibres plus clairsemées, recouvrant la glande malaire inférieure qu'on devine au travers de cette nappe semitransparente (Lesbre au contraire, affirmait que cette glande recouvrait le tendon du muscle.

Par ailleurs, la ligne d'insertion des fibres postérieures est plus étroite que celle des fibres antérieures, les tendons sont minces et déliés, de sorte que les glandes buccales les font saillir en haut comme en bas : en dehors de la ligne d'insertion, la région forme donc une bordure épaisse et saillante.

En avant, la couche profonde du buccinateur est peu développée et se disloque vite au contact des glandes buccales.

D'après cette description, le buccinateur affecte une forme évasée en entonnoir; la portion élargie est située en avant et permet à la bouche de s'ouvrir amplement.

Lesbre fait mention d'un muscle confondu avec le risorius, dont les fibres s'élèvent de la mandibule à la commissure des lèvres.

Bien que ce muscle prenne naissance à la mandibule comme l'affirme T,esbre, sal couche externe apparaît détachée du buccinateur. Ce serait plutôt un muscle résumant. l'action de l'incisif inférieur et de l'abaisseur de la lèvre inférieure du choval (co dernier, dans tue faible mesure).

Le mental est moyennement développé. En arrière, il se confond avec le précédent par sun origine.

Le dilatateur des narines fait défaut.

Le nasal : sa portion ventrale est bien développée (Lesbre dit que le nasal fait défaut). Il prend origine sur le bord libre de l'intermaxillaire et sur son apophyse nasale. Quelques-unes de ses fibres se confondent avec les muscles de la lèvre supérieure: elles semblent abaisser la lèvre. supérieure.

\section{Muscles des paupières.}

L'orbiculaire des paupières est un sphincter large et épais s'unissant en bas au muscle malaire.

Le frontal (de Lesbre) (corrugator supercilii) est bien développé en tant que corrugator supercilii mais ce n'est pas un frontal dans le sens où l'entend. Lesbre.

\section{Muscles des mâchoires.}

Le masséter est moins développé que chez le cheval. Ses fibres recouvrent la dernière molaire. Il rappelle le masséter du cheval; mais les fibres 
postérieures de la couche superficielle sont dirigées obliquement et s'incurvent de haut en bas et d'avant en arrière, pour s'insérer à l'angle du maxillaire inférieur. Ce sont les seules fibres insérées à ce niveau.

Le ptérygoidien interne. - Son origine est très étendue par suite de l'ampleur de la partie verticale de l'os palatin qui prend part à la constitution de l'orbite. naissance au niveau de la face externe de la lame verticale du palatin, bordé en avant et en bas par l'origine du muscle précédent et sur l'arcade ptérygoïdienne (représentant le conduit ptérygoïdien du cheval). En arrière, le muscle perd sa forme aplatie pour devenir épais et cylindrique et s'insère sur la face interne du condyle du maxillaire inférieur et le disque articulaire. Ainsi, ce muscle produit des mouvements latéraux accentués, parce que son

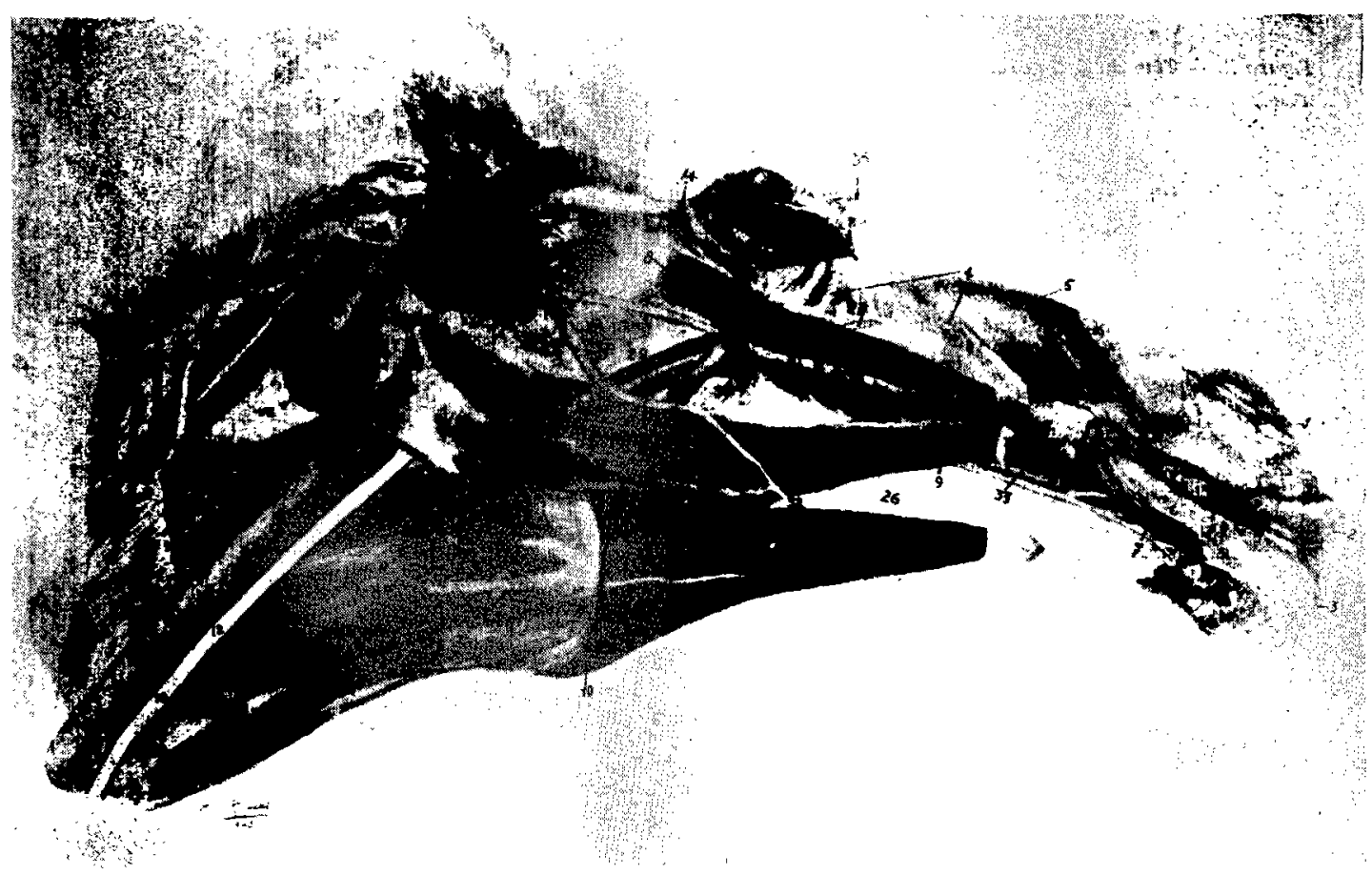

Figure I. - Tête du Chameau.

PJan superficiel.

1. Narine. - 2. Lèrre supérieure. - 3. Lèvre inférieure. 4. Malaire. - 5. Releveur commun de l'aile du nez et de la lèvre supérieure. - 6. Buccinateur. - 1. Orbiculaire des lèvres. - 8. Muscle zygomatique. - 9. Risorius. - 10. Mylohyoïlien. - 11. Omo-hyroidien. - 12. Veine jugulaire. -

Le ptérygoïdien interne se détache donc de la face externe de cette lame osseuse; les longues fibres antérieures naissent du plancher de l'orbite à proximité du conduit optique; vers l'arrière, les fibres diminuent progressivement d'ampleur, de sorte que l'insertion de ce muscle se fait suivant une ligne demi-circulaire qui s'éteint à l'extrémité inférieure de l'os ptérygoidien. Par suite du voisinage de cette attache avec le plan médian, les mouvements de diduction de la mâchoire sont très amples chez le chameau.

Le ptérygoìdien externe. - Ce muscle est bien délimité (Lesbre affirme qu'il n'est pas distinct). Il prend
13. Grand droit antérieur de la tête. - 14. Orbiculaire des paipières. - 15. Complexus. - 18. Parotido-auriculaire. 19. Sterno-mastoídien (sterno-céphalique). - 20. Sterno-thyroidien (le sterno-hyoidien fail défaut chez le chameau). 21. Glande sous-marillaire. - 22. Parotide. - 23 et 24 . Ganglions parondiens. - 25. Zygomato-auriculaire. - 26. Mâchoire inférjeure. -- 29. Canal de Sténon.

irserion est plus proche du plan médian que son oriqine.

L'occipito-mandibulaire. - Ce muscle est plus court que celui du cheval, son tendon supérieur se confond avec l'origine du digastrique. Inférieurement, la discrimination entre les deux muscles est pilutôt délicate.

Le digastrique. - Le tendon intermédiaire de ce muscle est épais, aplati, mi-charnu, mi-tendineux, absolument libre par rapport au stylo-hyoïdien.

Le digastrique est court, mais épais.

Les muscles hyoidiens : rien' à ajouter aux travaux de Lesbre. 


\section{Les muscles auriculaires.}

Un examen de la conque révèle ses petites dimensions; son ouverture est externe. Son grand axe mesure 16 centimètres environ; il coïncide avec celui de la tête. $L_{\imath}$ extrémité forme une pointe mousse
La plupart des muscles auriculaires rappellent ceux du cheval, hormis quelques différences.

Le scutellaire ressemble à celui du cheval, mais il est plus épais.

Le zygomato-auriculaire est relativement court.
Figure II. - Tête du Chameau. Plan superficiel. Face dorsale.

1. Cervico-auriculare.

1.. Son insertion.

2. Scutellaire partiellement sectionné pour montrer le crotaphite.

3. Cervico-ăuriculaire profond supérieur.

4. Pariéto-auriculaire.

5. Scuto.auriculaire superficiel inférieur.

6. Ganglion Iymphatique.

7. Cartilage scutiforme.

9. Crâte pariétale.

10. Frontal.

11. Tendon d'origine du releveur commun de l'aile du nez et de la lèvre supérieure.

12. Glande occipitale.

22. Complezus.

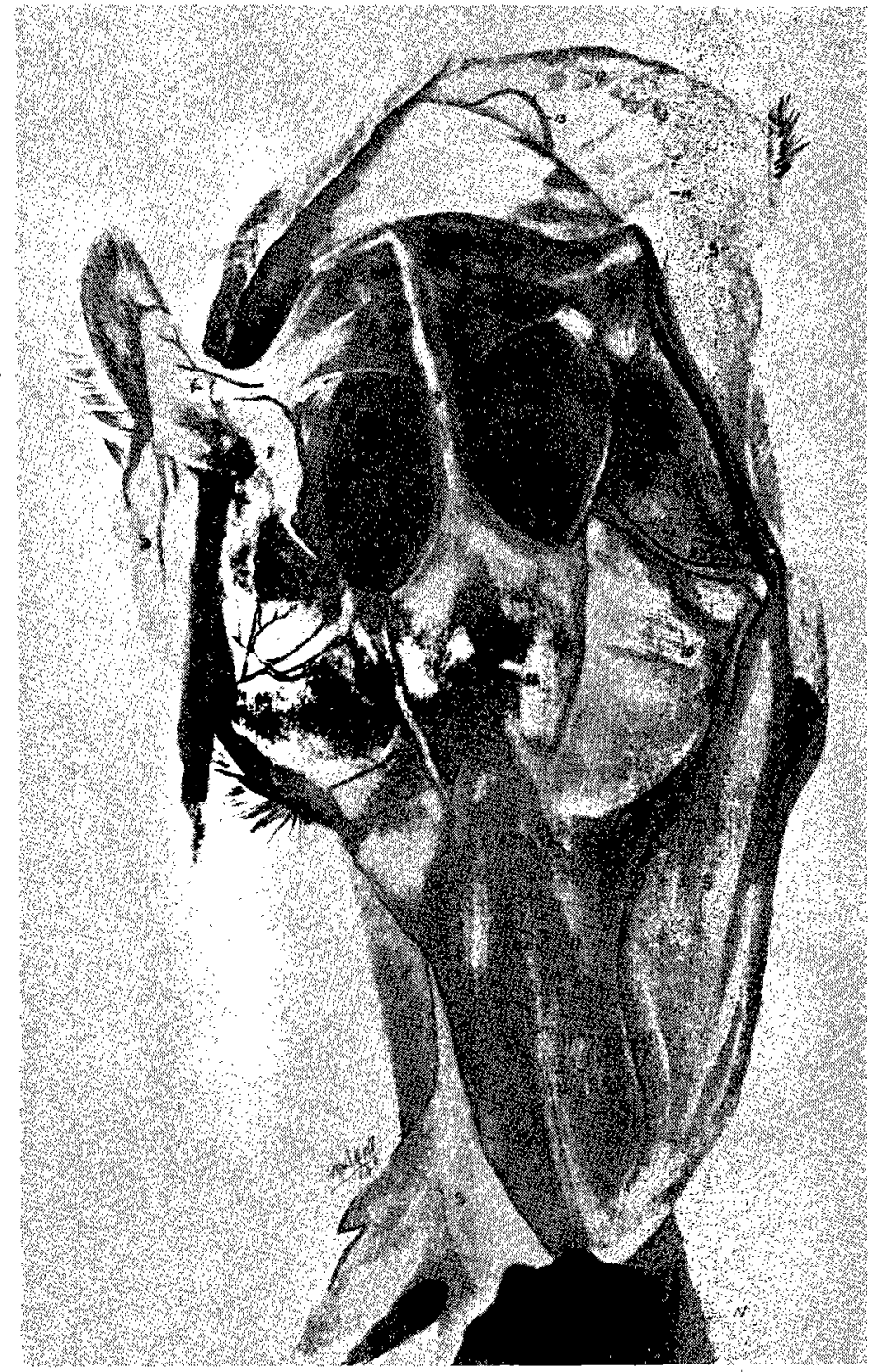

et sa base est beaucoup moins convexe que chez le cheval. Elle est recouverte de longs poils dans toute son étendue.

Le cartilage scutiforme est de forme quadrilatère, de dimensions 6-7 centimètres sur 4-5 centimètres. Son bord supérieur est échancré pour s'adapter à la conque. Le cartilage annulaire est petit.
Le scuto-auriculaire superficiel inférieur est plus développé.

Le scuto-auriculaire superficiel moyen est absent. Le scuto-auriculaire superficiel supérieur ne présente rien de particulier.

Le scuto-auriculaire superficiel accessoire n'existe pas.

Le pariéto-auriculaire est épais, triangulaire, 
s'élève de la crête pariètale, se dirige en denors pour s'attacher en partie inférieure du côté interne de la conque, recouvert par le cervico-auriculaire superficiel.

Le cervico-auriculaire superficiel ne presente rien de particulier.

Le cervico-auriculaire moyen s'élève de la crête pariétale (du même point que le temporo-aur:culaire) et de la protubérance occipitale. Ce long muscle triangulaire s'étend en dehors et en arrière pour s'insérer en partie postérieure de la base de la conque.

Le cervico-auriculaire profond. - Il est present, contrairement à ce que dit Lesbre.

Il est allonge, plus épais que celui du cheval.

Il se situe au bord postérieur de la protubérance occipitale (qui est très élevée chez le chameau). Il prend origine sur ce bord et sur le ligament cervical: il contourne latéralement la protubérance occipitale puis longe la partie postéro-externe de la conque pour s'insérer immédiatement au-dessous de l'ouverture conchinienne.

Le parotido-auriculaire est très petit et n'a que 2 à 3 centimetres de longueur.

Les rotateurs de l'oreille sont moins développés que chez le cheval.

Le muscle du tragus existe (contrairement à l'assertion de Lesbre).

\section{Les muscles de la région cervicale supéro. antérieure.}

Seule l'insertion du $\mathrm{m}$. complexus a été étudiée. Il en est de même pour les muscles droits antérieurs. Les autres muscles rappeilent ceux du cheval. Rien à dire également sur les muscles droits de la partie inférieure de la tête.

Le sterno-céphalique. - Ce muscle se termine d'une manière particulière; la couche exterre de ce muscle se confond avec celle qui dérive du muscle omo-ryoidien et forme le risorius déjà mentionné.

La partie interne du sterno-céphalique prend l'aspect d'un tendon arrondi très délié s'engageant ertre la glande sous-maxillaire et la parotide pour dégénérer avant d'atteindre l'apophyse mastoïde de l'os pétreux ou si elle l'atteint, se terminer par une aponévrose extrêmement mince à son contact.

\section{RÉSUMÉ}

Les muscles de la tête du chamcau ont ćté ćtudićs sur dix pièces injectées provenant de chameaux mâles et femelles, soudanais, prélevées aux abattoirs du Caire.

1. Il existe, chez le chameau, un risorius étendu, couvrant le maxillaire inférieur et l'angle de la mâchoire, formé par la couche externe du sternocéphalique confondue avec le peaussier qui se dégage de la surface de l'omo-hyoïdien.

2. Le releveur commun de la lèvre supérieure et de l'aile du nez n'est pas transpercé par le canin. Il se confond en arrière avec le muscle malaire qui sorme un vaste sus-naso-labial.

3. L'orbiculaire des lèvres forme un anneau incomolet, interrompu au milieu de la lèvre supérievire.

4. Le buccinateur est évasé en avant et permet d'oxvir largement la bouche.

5. Le releveur propre de la lèvre supérieure fait défaut et est remplacé dans une certaine mesure par ie canin.

6. Le pterygoïdien interne est très bien développé, tres raste et ses fibres sont disposées de façon à permetire d'anples mouvements de diduction de la mâchoire.

I. Le ptérygoïdien externe est bien développé, a:stinct du precédent et le renforce dans son action. 